\title{
Uncertainty Analysis in Transcranial Magnetic Stimulation Using Nonintrusive Polynomial Chaos Expansion
}

\author{
Konstantin Weise ${ }^{1}$, Luca Di Rienzo ${ }^{2}$, Hartmut Brauer ${ }^{1}$, Jens Haueisen ${ }^{3}$, and Hannes Toepfer ${ }^{1}$ \\ ${ }^{1}$ Department of Advanced Electromagnetics, Technische Universitaet Ilmenau, Ilmenau 98693, Germany \\ ${ }^{2}$ Dipartimento di Elettronica, Informazione e Bioingegneria, Politecnico di Milano, Milan 20133, Italy \\ ${ }^{3}$ Institute of Biomedical Engineering and Informatics, Technische Universitaet Ilmenau, Ilmenau 98693, Germany
}

\begin{abstract}
We propose a framework of nonintrusive polynomial chaos methods for transcranial magnetic stimulation (TMS) to investigate the influence of the uncertainty in the electrical conductivity of biological tissues on the induced electric field. The conductivities of three different tissues, namely, cerebrospinal fluid, gray matter (GM), and white matter, are modeled as uniformly distributed random variables. The investigations are performed on a simplified model of a cortical gyrus/sulcus structure. The statistical moments are calculated by means of a generalized polynomial chaos expansion using a regression and cubature approach. Furthermore, the results are compared with the solutions obtained by stochastic collocation. The accuracy of the methods to predict random field distributions was compared by applying different grids and orders of expansion. An investigation on the convergence of the expansion showed that in the present framework, an order 4 expansion is sufficient to determine results with an error of $<1 \%$. The results indicate a major influence of the uncertainty in electrical conductivity on the induced electric field. The standard deviation exceeds values of $20 \%-40 \%$ of the mean induced electric field in the GM. A sensitivity analysis revealed that the uncertainty in electrical conductivity of the GM affects the solution the most. This paper outlines the importance of exact knowledge of the electrical conductivities in TMS in order to provide reliable numerical predictions of the induced electric field. Furthermore, it outlines the performance and the applicability of spectral methods in the framework of TMS for future studies.
\end{abstract}

Index Terms - Eddy current, finite-element method (FEM), Monte Carlo (MC) method, regression analysis, sensitivity analysis, statistical analysis, stochastic processes, transcranial magnetic stimulation (TMS), uncertain systems.

\section{INTRODUCTION}

$\mathbf{T}$ RANSCRANIAL magnetic stimulation (TMS) is a non-invasive technique to stimulate cortical regions of the human brain by the principle of electromagnetic induction [1], [2]. In biphasic stimulation, a time-changing current with sinusoidal waveform is driven through an excitation coil. Consequently, an electric field is induced inside the human brain due to Faraday's law. The design and optimization of TMS coils involves simplifications to spherical head models to reduce the computational cost of iterative algorithms [3]. However, subsequent validation based on realistic head models derived from magnetic resonance images have to be performed to confirm the optimization results [4]. The complex geometry of the brain requires the application of numerical techniques, such as the finite-element method (FEM), in order to compute the spatial distribution of the induced electric field [5].

Thus, any kind of simulation study related to transcranial electromagnetic stimulation (not limited to TMS) requires exact knowledge of the corresponding electrical conductivity of the tissues in the head. However, in vivo measurements are difficult to obtain and vary between subjects due to individual factors, such as the cognitive state or baseline hormone levels. Hence, available data are widespread and exact individual predictions seem to be impossible [6], [7]. For that reason,

Manuscript received July 3, 2014; revised December 30, 2014; accepted January 7, 2015. Date of publication January 12, 2015; date of current version June 26, 2015. Corresponding author: K. Weise (e-mail: konstantin.weise@tu-ilmenau.de). an analysis regarding the uncertainty of the induced electric field in TMS appears imperative. Sampling methods, such as Monte Carlo (MC) approaches, are not applicable due to their slow convergence and the need for a large number of sampling points $\left(10^{4}-10^{6}\right)$. For that reason, we propose to apply a generalized polynomial chaos (gPC) expansion based on spectral projection or stochastic collocation (SC). Recent studies in this framework emphasize the importance of uncertainty quantification [8].

\section{Methods}

\section{A. Deterministic FEM Model}

A simplified geometry of a cortical gyrus/sulcus structure was used, similar to the one in [9] and [10]. The model is shown in Fig. 1(a). The coil is positioned at a distance of $10 \mathrm{~mm}$ above the volume conductor, which consists of a cerebrospinal fluid (CSF) layer with $20 \mathrm{~mm}$ thickness and an artificial sulcus with gray matter (GM) and white matter (WM). The sulcus has a depth of $21 \mathrm{~mm}$ and the GM layer has a thickness of $3 \mathrm{~mm}$.

A commercial figure-of-8 coil (Second Generation Double 70 mm - 3191-00, Magstim Company Ltd., Whitland, U.K.) acts as the excitation coil, assuming a current amplitude of $5488 \mathrm{~A}$ and a frequency of excitation of $2.9 \mathrm{kHz}$. The coil is positioned such that the normal component of the induced current density $\mathbf{J}$ is maximum, i.e., the long axis of the coil is parallel to the artificial sulcus. Due to the validity of the quasi-static approximation in TMS, the magnetic field produced by the coil is calculated in advance by means of the magnetic vector potential $\mathbf{A}$. The induced electric field is given by $\mathbf{E}=-j \omega \mathbf{A}-\nabla \varphi$. Commercial FEM software is used to determine the scalar 


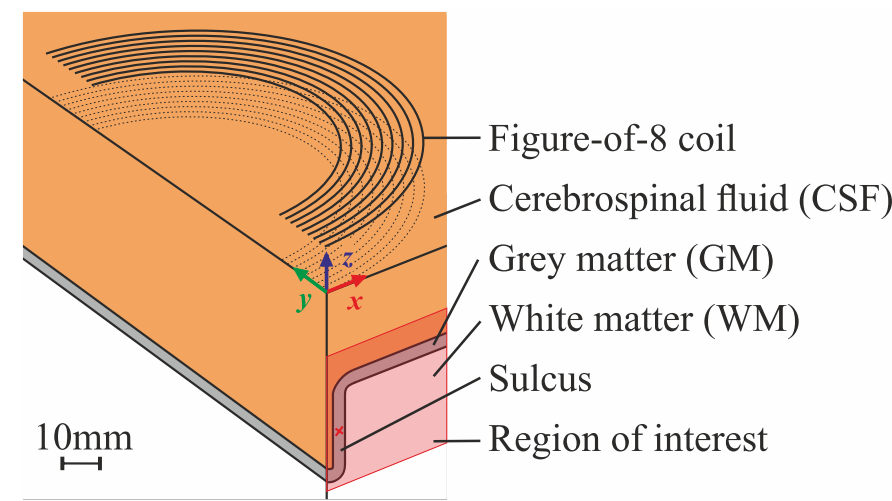

(a)

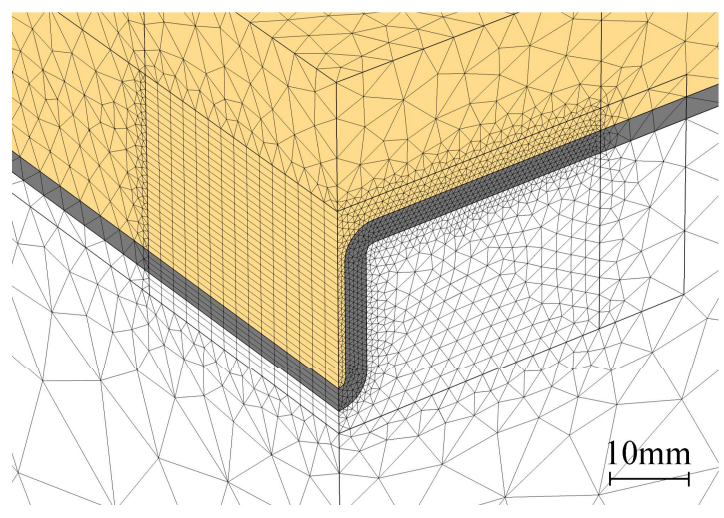

(b)

Fig. 1. (a) Quarter-model. (b) FEM mesh of the investigated cortical gyrus/sulcus structure. Red area and red cross: region of interest and a point inside GM where the pdf of the magnitude of the induced electric field is evaluated exemplarily.

electric potential $\varphi$ [11]. The FEM model shown in Fig. 1(b) makes use of the two symmetry planes $x z$ and $y z$ to further reduce computational costs resulting in only one quarter of the full geometry.

\section{B. Non-Intrusive Generalized Polynomial Chaos Expansion}

A gPC expansion can be conducted by either an intrusive or a nonintrusive approach. Intrusive approaches are based on Galerkin methods and imply a reformulation of the governing equations [12]-[14]. These approaches are usually memory intensive compared with nonintrusive approaches that make use of a distinct set of forward simulations to determine the stochastic properties of the output variables. In the present framework, we will focus on nonintrusive approaches and their application to TMS.

Considering the available data for CSF, GM, and WM, a wide range of electrical conductivity can be observed [5], [15]-[21]. Since the statistical properties of the tissues are not known, they are modeled as independent and uniformly distributed random variables with the following limits: 1) $1.4341 \leq \sigma_{\mathrm{CSF}} \leq 1.9487 \mathrm{~S} / \mathrm{m}$; 2) $0.1224 \leq \sigma_{\mathrm{GM}} \leq$ $0.5106 \mathrm{~S} / \mathrm{m}$; and 3) $0.0957 \leq \sigma_{\mathrm{WM}} \leq 0.1663 \mathrm{~S} / \mathrm{m}$. They are combined in a three-variate random vector $\xi(N=3)$ located in the probability space $(\Xi, \Sigma, P)$. The event space $\Xi$ contains all possible events, $\Sigma$ is a $\sigma$-algebra, which is a subset of $\Xi$, and $P$ is a measure for the probability of occurrence. Based on this, the gPC for uniformly distributed random variables is defined by a truncated expansion of Legendre polynomials $\psi_{k}(\xi)$ weighted by the gPC coefficients $\hat{u}_{k}$ [22]

$$
E(\mathbf{r}, \boldsymbol{\xi})=\sum_{k=0}^{\infty} \hat{u}_{k}(\mathbf{r}) \psi_{k}(\boldsymbol{\xi}) \approx \sum_{k=0}^{N_{c}-1} \hat{u}_{k}(\mathbf{r}) \psi_{k}(\boldsymbol{\xi}) .
$$

In the present framework, the output $E(\mathbf{r}, \boldsymbol{\xi})$ is the magnitude of the induced electric field at a certain point $\mathbf{r}$. In the case of a maximum order gPC expansion with order $p$, the total number of coefficients $N_{c}$ in the case of $N$ random variables is given by [23]

$$
N_{c}=\left(\begin{array}{c}
N+p \\
N
\end{array}\right)=\frac{(N+p) !}{N ! p !} .
$$

In the following, two numerical methods are presented to determine the gPC coefficients, namely: 1) the spectral projection approach by Gauss-Legendre quadrature and 2) the regression approach.

1) Spectral Projection by Gauss-Legendre Quadrature: Due to the orthogonality of the polynomial basis functions $\psi_{k}(\boldsymbol{\xi})$, the gPC coefficients $\hat{u}_{k}(\mathbf{r})$ can be determined by [24]

$$
\hat{u}_{k}(\mathbf{r})=\frac{\left\langle E(\mathbf{r}, \boldsymbol{\xi}), \psi_{k}(\boldsymbol{\xi})\right\rangle}{\left\langle\psi_{k}(\boldsymbol{\xi}), \psi_{k}(\boldsymbol{\xi})\right\rangle}, \quad k=0,1, \ldots, N_{c}-1 .
$$

The denominator in (3) acts as a scalar weight for each coefficient and can be calculated analytically. However, the numerator has to be calculated numerically, since the function $E(\mathbf{r}, \xi)$ is not known. Depending on the probability density function (pdf) of the input variables, this integral can be solved by means of a distinct type of Gauss quadrature. In the present case of standard uniform distributions in the interval $[-1,1]$, a Gauss-Legendre quadrature is applied

$$
\begin{aligned}
\left\langle E(\mathbf{r}, \boldsymbol{\xi}), \psi_{k}(\boldsymbol{\xi})\right\rangle= & \int_{-1}^{+1} E(\mathbf{r}, \boldsymbol{\xi}) \psi_{k}(\boldsymbol{\xi}) 0.5^{N} d \boldsymbol{\xi} \\
\approx & \sum_{i_{1}=1}^{m_{1}} \cdots \sum_{i_{N}=1}^{m_{N}} E\left(\mathbf{r}, \xi_{1}^{\left(i_{1}\right)}, \ldots, \xi_{N}^{\left(i_{N}\right)}\right) \\
& \times \psi_{k}\left(\xi_{1}^{\left(i_{1}\right)}, \ldots, \xi_{N}^{\left(i_{N}\right)}\right) w_{i_{1}}^{\left(m_{1}\right)}, \ldots, w_{i_{N}}^{\left(m_{N}\right)}
\end{aligned}
$$

where $n=(1,2, \ldots, N)$ denotes the respective dimension, $m_{n}$ is the number of cubature points in the $n$th dimension, and $w_{i_{n}}^{\left(m_{n}\right)}$ is the corresponding weight.

According to the uniform distribution of the input, the grid points are chosen as the roots of the Legendre polynomials [24]. In this paper, the grid is 3-D. It is calculated by means of the tensor product of the roots given by the 1-D Legendre polynomials of degree $m$, resulting in $m^{3}$ nodes.

2) Regression Approach: The regression approach employs a least square solution in order to determine the $\mathrm{gPC}$ coefficients

$$
\Psi \hat{\mathbf{u}}(\mathbf{r})=\mathbf{s}(\mathbf{r}) .
$$

The matrix $\Psi$ is of size $\left[N_{d} \times N_{c}\right]$, with $N_{d}$ denoting the number of deterministic forward simulations. Each row 
contains the $N_{c}$ multivariate polynomials $\psi_{k}$ calculated at the sample points $\xi_{i}$ with $i=1, \ldots, N_{c}$. The unknown vector $\hat{\mathbf{u}}(\mathbf{r})$ of size $\left[N_{c} \times 1\right]$ contains the gPC coefficients $\hat{u}_{k}(\mathbf{r})$, which correspond to each polynomial $\psi_{k}(\xi)$. The calculated deterministic forward solutions at the grid points $\boldsymbol{\xi}_{i}$ are composed in the vector $\mathbf{s}(\mathbf{r})$ of size $\left[N_{d} \times 1\right]$ on the right-hand side. The Moore-Penrose pseudo-inverse based on singular value decomposition is used to determine the gPC coefficients. The same tensor product-based Legendre grid as described previously is applied for the regression approach.

\section{Stochastic Collocation by Lagrange Interpolation}

The SC approach in the framework of uncertainty analysis was first introduced in [25]. In contrast to previously presented methods, the SC is not based on the projection of the solution on the random space. Instead, the solution is approximated by a set of multivariate Lagrange interpolation functions. This set contains one polynomial for each collocation point. The advantage of Lagrange interpolation is that the associated function equals 1 in the corresponding collocation point and 0 in all other points. Thus, the approximation is exact at the collocation points and smoothly interpolated in between adjacent points. The polynomial for an $N$-dimensional interpolation can be constructed by tensor products of 1-D Lagrange polynomials $\mathcal{L}_{i}^{\left(m_{n}\right)}(\xi)$ [24]

$$
\mathcal{L}_{i}^{m_{1}}(\xi)=\prod_{\substack{j=1 \\ j \neq i}}^{m_{1}} \frac{\xi-\xi_{j}}{\xi_{i}-\xi_{j}} .
$$

The global interpolation function can then be constructed by the principle of superposition

$$
\begin{aligned}
E(\mathbf{r}, \boldsymbol{\xi}) \approx & \sum_{i_{1}=1}^{m_{1}} \ldots \sum_{i_{N}=1}^{m_{N}} E\left(\mathbf{r}, \xi_{1}^{\left(i_{1}\right)}, \ldots, \xi_{N}^{\left(i_{N}\right)}\right) \\
& \times \mathcal{L}_{i_{1}}^{\left(m_{1}\right)}\left(\xi_{1}\right), \ldots, \mathcal{L}_{i_{N}}^{\left(m_{N}\right)}\left(\xi_{N}\right) .
\end{aligned}
$$

During implementation of the SC, it proven to be useful for postprocessing to reformulate (8) into the form like that used in $\mathrm{gPC}(1)$

$$
E(\mathbf{r}, \boldsymbol{\xi}) \approx \sum_{k=0}^{N_{l}-1} \hat{l}_{k}(\mathbf{r}) \mathcal{L}_{k}(\xi)
$$

where $N_{l}$ denotes the number of Lagrange coefficients $\hat{l}_{k}(\mathbf{r})$, which is equivalent to the number of grid points $N_{d}$. The function $\mathcal{L}_{k}(\xi)$ is the Lagrange interpolation polynomial at the corresponding point indexed by $k$.

Since the nonuniformity of the nodal distribution improves the quality of Lagrange interpolation [22], the same grid as was used in the case of Gauss quadrature is applied in SC. This results in a total number of $N_{l}=m^{3}$ terms in (9), whereas the maximum order of each random variable is $m-1$.

\section{Postprocessing}

After calculating the gPC coefficients $\hat{u}_{k}$ by means of Gauss quadrature or regression, the statistical moments can be determined. Using an indexation such that $\psi_{0}=1$, the mean $E_{\mu}(\mathbf{r})$ and the variance $E_{\sigma^{2}}(\mathbf{r})$ of the induced electric field $E(\mathbf{r}, \boldsymbol{\xi})$ are given by

$$
\begin{aligned}
E_{\mu}(\mathbf{r}) & =\mathbb{E}[E(\mathbf{r}, \boldsymbol{\xi})]=\psi_{0} \mathbb{E}[E(\mathbf{r}, \boldsymbol{\xi})] \\
& =\sum_{k=0}^{N_{c}-1} \hat{u}_{k} \int_{-1}^{+1} \psi_{0} \psi_{k}(\boldsymbol{\xi}) 0.5^{N} d \boldsymbol{\xi}=\hat{u}_{0}(\mathbf{r}) \\
E_{\sigma^{2}}(\mathbf{r}) & =\mathbb{E}\left[\left(E(\mathbf{r}, \boldsymbol{\xi})-E_{\mu}(\mathbf{r})\right)^{2}\right] \\
& =\sum_{k=1}^{N_{c}-1} \hat{u}_{k}^{2} \int_{-1}^{+1} \psi_{k}(\boldsymbol{\xi})^{2} 0.5^{N} d \xi
\end{aligned}
$$

Hence, the mean is directly given by the first $\mathrm{gPC}$ coefficients due to the orthogonality of the gPC basis functions with respect to the pdf. The variance can be determined by the remaining coefficients, whereas the integral in (11) can be calculated analytically. In the case of SC, the statistical moments can be calculated in a similar way. However, the Lagrange interpolation polynomials are not necessarily orthogonal, which leads to analytical integrations when employing (10) and (11).

The pdf can be determined by applying sampling strategies, such as MC, Latin Hypercube Sampling [26], or quasi-MC [27], [28] with $M$ realizations. Since a functional dependence is calculated by either the $\mathrm{gPC}$ or $\mathrm{SC}$, a large number of samples, such as $M \approx 10^{5}-10^{6}$, can be used with a significantly decreased computational effort compared with direct sampling with the FEM model.

An important part during the investigation of new or partially known systems is an analysis regarding its sensitivity. In gPC, the global derivative-based sensitivity coefficients $S_{n}$ can be approximated by means of the gPC coefficients and the partial derivatives of the basis functions [29]. In this context, the index $n$ denotes the materials CSF, GM, or WM

$$
\begin{aligned}
S_{n}(\mathbf{r}) & =\mathbb{E}\left[\frac{\partial E(\mathbf{r}, \boldsymbol{\xi})}{\partial \xi_{n}}\right] \\
& \approx \sum_{k=0}^{N_{c}-1}\left(\hat{u}_{k}(\mathbf{r}) \int_{-1}^{+1} \frac{\partial \psi_{k}(\boldsymbol{\xi})}{\partial \xi_{n}} 0.5^{N} d \boldsymbol{\xi}\right) .
\end{aligned}
$$

Due to the reformulation of the Lagrange interpolation function in (9), the same formula can be applied to determine the sensitivity coefficients in the case of SC by replacing $\hat{u}_{k}(\mathbf{r})$ and $\psi_{k}(\xi)$ with $\hat{l}_{k}(\mathbf{r})$ and $\mathcal{L}_{k}(\xi)$, respectively.

\section{E. Error Estimation}

It is shown that SC yields exact results at the collocation points. However, the interpolation in between grid points might be inaccurate, especially when considering higher dimensions. To evaluate the accuracy of gPC (regression and quadrature) and SC (Lagrange interpolation), the results were compared with an independent test set [30]. This set contains $M^{\prime}$ randomly selected points $\boldsymbol{\xi}^{\prime}=\left\{\boldsymbol{\xi}_{(1)}^{\prime}, \ldots, \boldsymbol{\xi}_{\left(M^{\prime}\right)}^{\prime}\right\}$ according to the pdfs of the input variables. They are not used to estimate the coefficients in gPC or SC. To obtain a global measure, the goodness of fit is determined in the domain of 
TABLE I

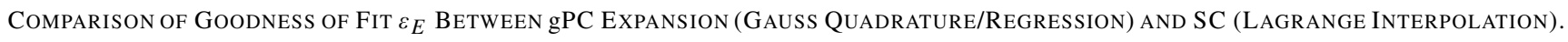

Double Entries SeParated by a Slash Symbol RePresent Goodness of Fit of Gauss QuAdrature and REgRESSION,

Respectively; $m$ Is Number of Grid Points in One Dimension, $N_{d}$ Is Total Number of Forward Simulations,

$N_{c}$ Is Number of gPC COEFFicients, and $p$ Is Order of ExPansion. Bold VALUes Indicate Best

Goodness of Fit Values at a Given Number of Forward Simulations. gPC CoefFicients

Were Determined UnTIL $N_{c} \leq N_{d}$ FOR REgRESSION AND $p \leq 2 m-1$ FOR QUADRATURE

\begin{tabular}{|c|c|c|c|c|c|c|}
\hline & $m$ & 3 & $\begin{array}{l}4 \\
64\end{array}$ & 5 & 6 & 7 \\
\hline & \multirow[t]{3}{*}{$N_{d}$} & 27 & 64 & 125 & 216 & 343 \\
\hline & & \multicolumn{5}{|c|}{ SC (Lagrange Interpolation) } \\
\hline & & .9030 & .9683 & .9897 & .9966 & .9988 \\
\hline$N_{c}$ & $p$ & \multicolumn{5}{|c|}{ gPC (Quadrature / Regression) } \\
\hline 10 & 2 & $.8947 / .8943$ & $.8991 / .8969$ & $.8991 / .8942$ & $.8991 / .8921$ & $.8991 / .8905$ \\
\hline 20 & 3 & $.9030 / .9029$ & $.9657 / .9655$ & $9670 / .9663$ & $.9670 / .9655$ & $.9670 / .9648$ \\
\hline 35 & 4 & $.7155 /-$ & . $9683 / .9683$ & $.9888 / .9888$ & $.9893 / .9892$ & $.9893 / .9890$ \\
\hline 56 & 5 & $-.0227 /-$ & .9080/.9372 & $.9897 / .9897$ & $.9963 / .9963$ & $.9965 / .9964$ \\
\hline 84 & 6 & $-1-$ & $.7099 /-$ & $.9700 / .9788$ & $.9966 / .9966$ & .9987 / .9987 \\
\hline 120 & 7 & $-1-$ & $-.0432 /-$ & $.9071 / .9542$ & $.9903 / .9930$ & $.9988 / .9988$ \\
\hline 165 & 8 & $-1-$ & $-1-$ & $.7075 /-$ & $.9699 / 0.9839$ & $.9968 / .9976$ \\
\hline 220 & 9 & $-1-$ & $-1-$ & $-.0456 /-$ & $.9074 /-$ & $.9904 / .9946$ \\
\hline 286 & 10 & $-1-$ & $-1-$ & $-1-$ & $.7082 /-$ & $.9701 / .9865$ \\
\hline 364 & 11 & $-1-$ & $-1-$ & $-1-$ & $-.0430 /-$ & $.9078 /-$ \\
\hline 455 & 12 & $-1-$ & $-1-$ & $-1-$ & $-1-$ & $.7100 /-$ \\
\hline 560 & 13 & $-1-$ & $-1-$ & $-1-$ & $-1-$ & $-.0519 /-$ \\
\hline
\end{tabular}

TABLE II

(a) Determinant of INFormation Matrix $\Psi^{T} \Psi$. (b) CONDition Number of gPC Matrix $\Psi$ For Different Tensored GRidS and Expansion Orders $p$. Bold Values Indicate Best Goodness of Fit Values From Table I at a Given Number of Forward Simulations. Determinants Were Determined Until $N_{c}<N_{d}$

\begin{tabular}{|c|c|ccccc|}
\cline { 2 - 7 } \multicolumn{1}{c|}{} & $m$ & 3 & 4 & 5 & 6 & 7 \\
\hline \multirow{2}{*}{$N_{c}$} & $N_{d}$ & 27 & 64 & 125 & 216 & 343 \\
\hline 10 & 2 & $3 \mathrm{E}+08$ & $6 \mathrm{E}+12$ & $8 \mathrm{E}+15$ & $2 \mathrm{E}+18$ & $3 \mathrm{E}+20$ \\
20 & 3 & $\mathbf{3 E}-127$ & $7 \mathrm{E}+20$ & $3 \mathrm{E}+27$ & $5 \mathrm{E}+32$ & $1 \mathrm{E}+37$ \\
35 & 4 & - & $\mathbf{5 E}-109$ & $1 \mathrm{E}+41$ & $4 \mathrm{E}+50$ & $2 \mathrm{E}+58$ \\
56 & 5 & - & 0 & $\mathbf{1 E - 8 2}$ & $1 \mathrm{E}+71$ & $9 \mathrm{E}+83$ \\
84 & 6 & - & - & 0 & $\mathbf{- 4 E}-41$ & $7 \mathrm{E}+112$ \\
120 & 7 & - & - & 0 & 0 & $\mathbf{1 E}+\mathbf{0 9}$ \\
165 & 8 & - & - & - & 0 & $7 \mathrm{E}-273$ \\
220 & 9 & - & - & - & - & 0 \\
286 & 10 & - & - & - & - & 0 \\
\hline
\end{tabular}

(a)

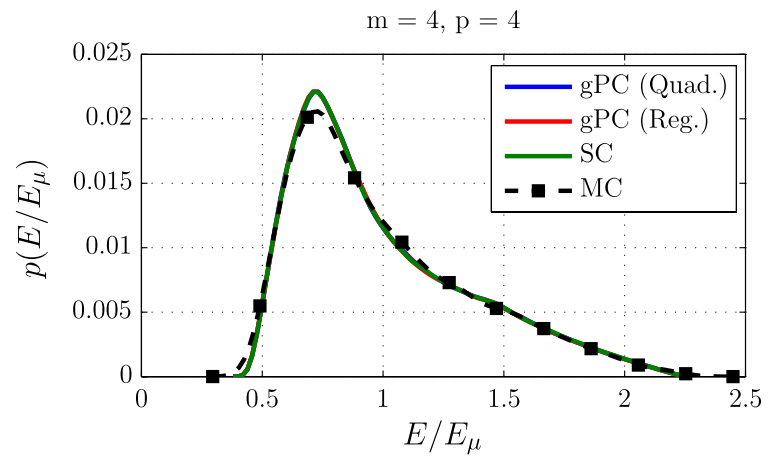

(a)

\begin{tabular}{|c|c|c|c|c|c|c|}
\hline & $m$ & 3 & 4 & 5 & 6 & 7 \\
\hline$N_{c}$ & $N_{p}$ & 27 & 64 & 125 & 216 & 343 \\
\hline 10 & 2 & $2 \mathrm{E}+00$ & $2 \mathrm{E}+00$ & $2 \mathrm{E}+00$ & $2 \mathrm{E}+00$ & $2 \mathrm{E}+00$ \\
\hline 20 & 3 & $2 \mathrm{E}+16$ & $3 E+00$ & $3 E+00$ & $3 E+00$ & $3 \mathrm{E}+00$ \\
\hline 35 & 4 & - & $2 \mathrm{E}+30$ & $5 \mathrm{E}+00$ & $5 E+00$ & $5 \mathrm{E}+00$ \\
\hline 56 & 5 & - & $2 \mathrm{E}+31$ & $4 E+31$ & $7 \mathrm{E}+00$ & $7 \mathrm{E}+00$ \\
\hline 84 & 6 & - & - & $9 \mathrm{E}+30$ & $2 \mathrm{E}+30$ & $9 \mathrm{E}+00$ \\
\hline 120 & 7 & - & - & $3 E+31$ & $6 \mathrm{E}+30$ & $2 E+31$ \\
\hline 165 & 8 & - & - & - & $5 E+30$ & $5 E+31$ \\
\hline 220 & 9 & - & - & - & - & $6 \mathrm{E}+30$ \\
\hline 286 & 10 & - & - & - & - & $1 \mathrm{E}+31$ \\
\hline
\end{tabular}

(b)

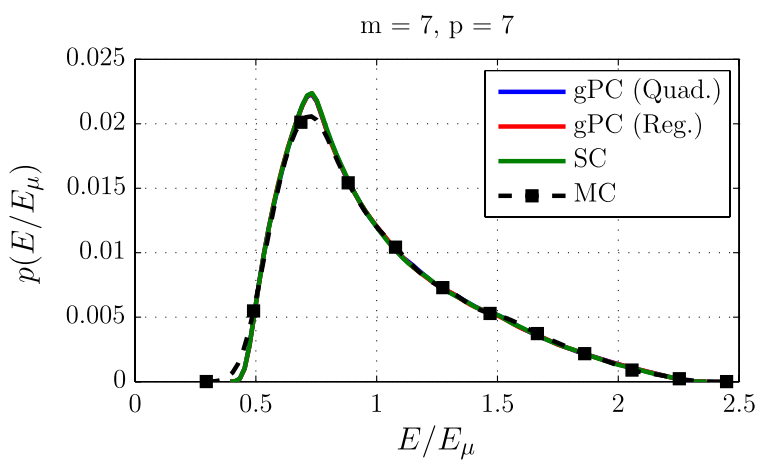

(b)

Fig. 2. Comparison of the normalized pdfs of the induced electric field between $\mathrm{gPC} / \mathrm{SC}$ and MC simulations at the point $(x, y, z)=(2.8,0,-31.1) \mathrm{mm}$ in case of (a) order $p=4$ approximation and (b) order $p=7$ approximation.

interest $\mathbf{r} \in \Omega^{\prime}$, which is defined in the $x z$ plane at $y=0 \mathrm{~mm}$. The results obtained by the expansions $\tilde{E}\left(\mathbf{r}, \xi^{\prime}\right)$ for these values are compared with the exact results $E\left(\mathbf{r}, \boldsymbol{\xi}^{\prime}\right)$ in the root mean square sense. Since the expansion is performed for every FEM-node in $\Omega^{\prime}$, an additional integration is performed over
$\Omega^{\prime}$ weighted by the total volume $V_{\Omega^{\prime}}$ to obtain a measure for the global error in this region

$$
\varepsilon_{E}=\frac{1}{V_{\Omega^{\prime}}} \int_{\Omega^{\prime}} 1-\frac{\left\|E\left(\mathbf{r}, \xi^{\prime}\right)-\tilde{E}\left(\mathbf{r}, \xi^{\prime}\right)\right\|}{\left\|E\left(\mathbf{r}, \xi^{\prime}\right)-\bar{E}\left(\mathbf{r}, \xi^{\prime}\right)\right\|} d \Omega .
$$




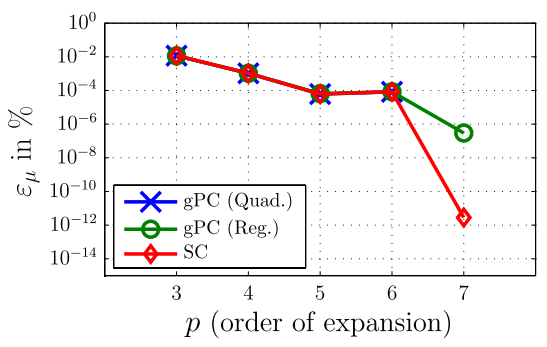

(a)

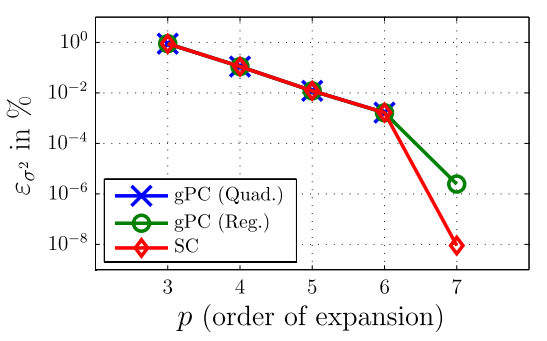

(b)

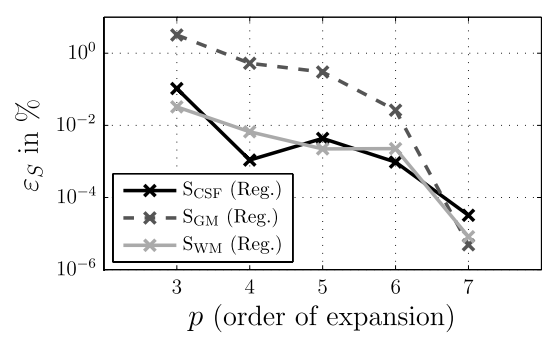

(c)

Fig. 3. gNRMSD $\varepsilon_{\mathrm{QOI}}$ from (14) in dependence of the expansion order $p$ for (a) mean value $\varepsilon_{\mu}$, (b) variance $\varepsilon_{\sigma^{2}}$, and (c) global sensitivity coefficients $\varepsilon_{S}$ of the induced electric field. All results are compared with an order $p=7(m=7)$ approximation determined by means of Gauss quadrature.

The variable $\bar{E}\left(\mathbf{r}, \boldsymbol{\xi}^{\prime}\right)$ denotes the mean of all $M^{\prime}$ samples. This error estimator can be understood as a modification to the second metric proposed in [31]. The disadvantage of this error estimator is an increase in computational cost to perform additional $M^{\prime}$ forward simulations. However, $\varepsilon_{E}$ quantifies the error of the approximation directly with respect to the output variable $E(\mathbf{r}, \boldsymbol{\xi})$. The estimates vary between $-\infty$ for bad fits and 1 in the case of perfect fits.

The convergence of the statistical moments and the sensitivity coefficients are evaluated by calculating the global normalized root mean square deviation (gNRMSD) with respect to the reference solution REF obtained by Gauss quadrature for an order $p=7$ expansion using $m=7$ points in each dimension

$$
\varepsilon_{\mathrm{QOI}}=100 \% \frac{\sqrt{\frac{1}{V_{\Omega^{\prime}}} \int_{\Omega^{\prime}}(\mathrm{QOI}(\mathbf{r})-\operatorname{REF}(\mathbf{r}))^{2} d \Omega}}{\max \operatorname{REF}(\mathbf{r})-\min \operatorname{REF}(\mathbf{r})}
$$

where $\mathrm{QOI}(\mathbf{r})$ is the quantity of interest. Hence, the gNRMSD of the mean and the variance are denoted as $\varepsilon_{\mu}$ and $\varepsilon_{\sigma^{2}}$, respectively.

\section{RESUlTS AND Discussion}

\section{A. Convergence of Expansion}

The goodness of fit is calculated for different grids by varying the number of grid points $m$ in each dimension. The results are shown in Table I for gPC and SC using a validation set of size $M^{\prime}=10^{4}$. We observed very similar results using a smaller validation set of $M^{\prime} \approx 10^{2}-10^{3}$ points (data not shown). Nevertheless, this sample set is also used to compare the pdfs obtained by MC with gPC and SC.

On every grid, the calculations are performed for different expansion orders $p$. For gPC, the highlighted values indicate the best combination between the grid discretization $m$ and the order of expansion $p$. It can be observed that an increased expansion order $p$ leads to better fits. Thus, nonlinear relationships between the induced electric field and the electrical conductivity are present, which result from the geometrical structure of the problem. When comparing the goodness of fits at one distinct grid, it can be observed that the error is minimized, when choosing $p=m$, i.e., the expansion order corresponds to the number of grid points in one dimension. On the one hand, when choosing $p<m$, each individual gPC coefficient is determined more accurately. However, the error increases due to truncating the expansion too early. On the

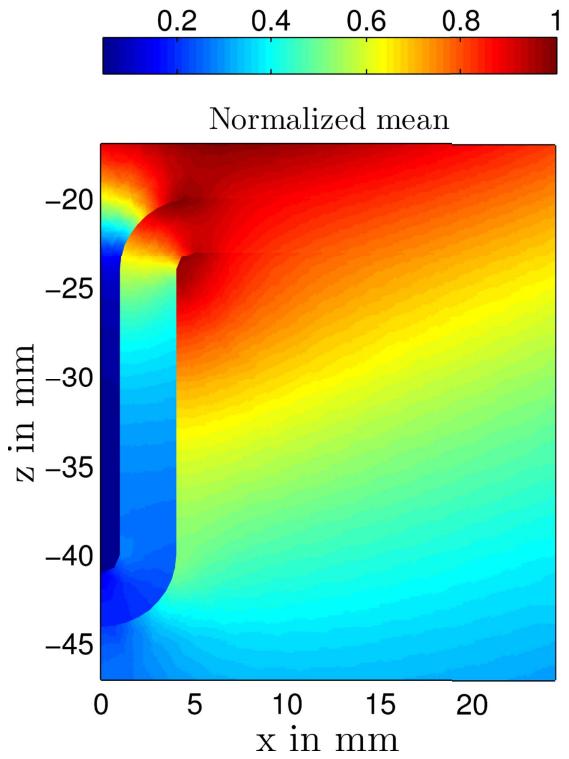

(a)

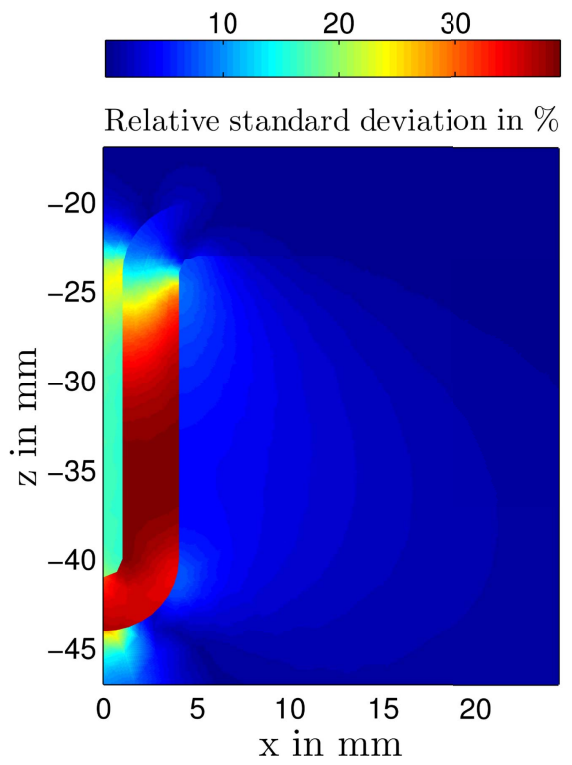

(b)

Fig. 4. Spatial distribution of (a) normalized mean and (b) relative standard deviation of the induced electric field under the figure-of- 8 coil in the $x z$ plane at $y=0 \mathrm{~mm}$ (Gauss quadrature, $p=7, m=7$ ).

other hand, when choosing $p>m$, the error increases because the number of deterministic forward simulations is too low, for both quadrature and regression. Considering the optimal 


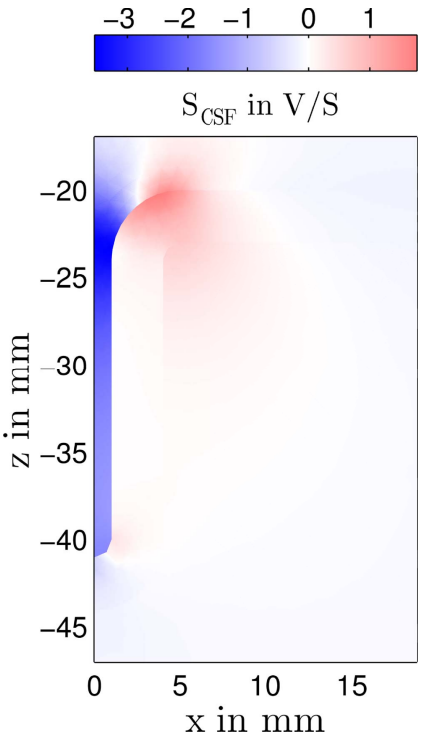

(a)

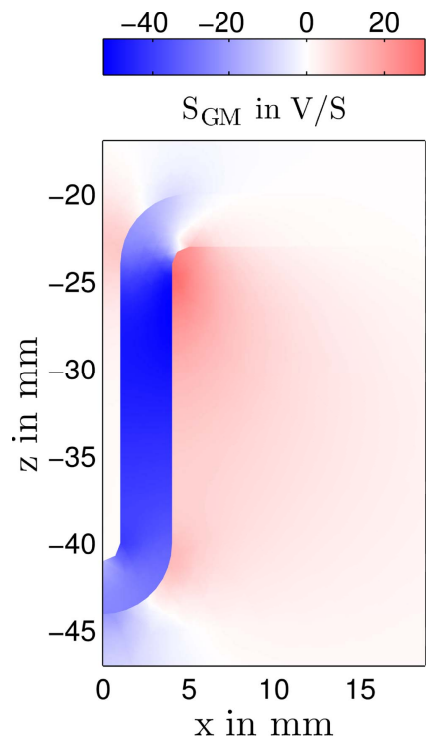

(b)

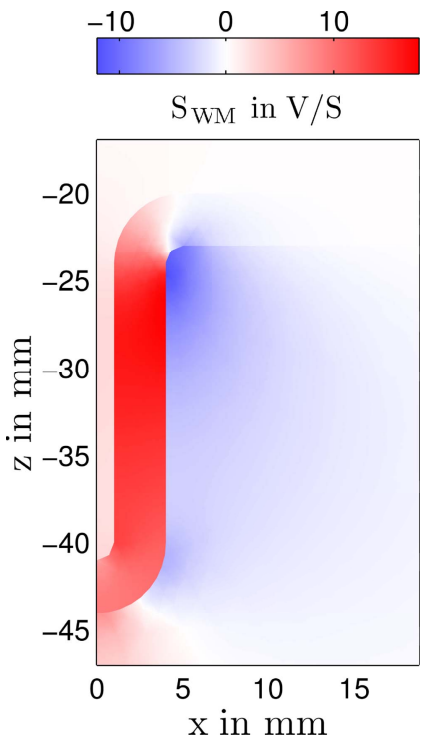

(c)

Fig. 5. Spatial distribution of the global sensitivity coefficients (a) $S_{\mathrm{CSF}}$, (b) $S_{\mathrm{GM}}$, and (c) $S_{\mathrm{WM}}$ in the $x z$ plane at $y=0 \mathrm{~mm}$ (Gauss quadrature, $p=7, m=7)$.

choices for $m$ and $p$, all methods perform almost equally well. In the case of regression, the ratio between the number of forward simulations and the number of coefficients increases for higher orders $p$.

The determinant of the information matrix $\Psi^{T} \Psi$ is shown in Table II(a). It is proportional to the generalized variance, which is a measure for multidimensional scatter in the resulting gPC coefficients [32]-[34].

The condition number of the gPC matrix $\Psi$, which is a measure of the output sensitivity with respect to small errors in the input $\hat{\mathbf{u}}(\mathbf{r})$, is shown in Table II(b).

A sharp transition can be observed between $p=m-1$ and $p=m$. This effect occurs when the overdeterminacy of the linear system is decreased at the point where the number of gPC coefficients is greater than two times the number of forward simulations $N_{c}>2 N_{d}$. The determinant and the condition number indicate that $p=m-1$ would lead to the best results. However, the use of the Moore-Penrose pseudoinverse significantly improves the results obtained by regression and leads to the highest goodness of fit values at $p=m$ even for very high condition numbers and small determinants.

The pdf is obtained by direct MC-sampling with $10^{4}$ points and by sampling the gPC expansion $\mathrm{E}(\mathbf{r}, \boldsymbol{\xi})$ from (1) with $10^{5}$ points. Fig. 2 shows the pdfs for an order $p=4$ and $p=7$ expansion at the point $(x, y, z)=(2.8,0,-31.1) \mathrm{mm}$ located close to the sulcus in the center of GM [see red cross in Fig. 1(a)]. Slight differences between gPC, SC, and MC can be observed at the maximum, which can be explained by an inadequate number of direct MC simulations of only $10^{4}$. An order 4 expansion with only 64 forward simulations already approximates the resulting pdf very well.

Fig. 3 shows the convergence of the gNRMSD calculated by (14) of the mean, the variance, and the sensitivity coefficients. The results of the quadrature method for $p=7$ and $m=7$ are used as reference values, since they are determined to be the most exact (see Table I). As expected, the mean converges faster than the variance. The convergence rate between the global sensitivity coefficients showed noteworthy differences. It can be seen that $S_{\mathrm{GM}}$ converges remarkably slower than $S_{\mathrm{CSF}}$ and $S_{\mathrm{WM}}$. However, an order $p=4$ expansion $\left(N_{d}=64\right)$ resulted in errors $<1 \%$ for all sensitivity coefficients.

\section{B. Statistical Moments}

The spatial distribution of the expected value and the standard deviation are shown in Fig. 4(a) and (b), respectively. The results are shown in the region of interest shown in Fig. 1(a). The mean-induced electric field is normalized with respect to the maximum value in the $x z$ plane. The results are obtained by the reference solution (Gauss quadrature, $p=7, m=7$ ). The mean of the induced electric field was normalized with respect to its maximum value in this region. The normalized mean-induced electric field exceeds values of 0.9 in several small regions in CSF, the gyrus crown in GM as well as in WM.

The spatial distribution of the standard deviation in Fig. 4(b) was normalized with respect to the corresponding mean value of the induced electric field and multiplied by 100 to determine a relative measure in percent. An area where the relative standard deviation exceeds $30 \%-40 \%$ can be observed in the GM region. However, a sharp transition to adjacent domains can be observed, where the relative standard deviation drops to $20 \%$ in CSF at $x=0 \mathrm{~mm}$ and $5 \%-10 \%$ in WM at $x=5 \mathrm{~mm}$. Hence, using the presented methodology, it is possible to identify the specific areas where the uncertainty of the input variables amplifies the spread of the output quantities.

\section{Sensitivity Analysis}

The spatial distributions of the global sensitivity coefficients $S_{\mathrm{CSF}}, S_{\mathrm{GM}}$, and $S_{\mathrm{WM}}$ are shown in Fig. 5 . These coefficients indicate how strong and at which point in space the 
induced electric field is affected by changes of the electrical conductivity of CSF, GM, and WM, respectively.

It can be observed that, close to the sulcus region, the sensitivity coefficient is negative in the corresponding domain, i.e., $S_{\mathrm{CSF}}$ and $S_{\mathrm{WM}}$ reach values of less than -3 and $-10 \mathrm{~V} / \mathrm{S}$ at $x=0 \mathrm{~mm}$ in CSF and $x=-5 \mathrm{~mm}$ in WM, respectively. However, $S_{\mathrm{GM}}$ clearly exceeds the latter two coefficients and the values of less than $-40 \mathrm{~V} / \mathrm{S}$ inside GM at $x=-2.5 \mathrm{~mm}$ are reached. In general, a negative sensitivity can be understood as an inverse proportionality. This can be explained by Ohm's law and the boundary condition for $\mathbf{J}$. An increase in conductivity leads to a decrease of the induced electric field in the corresponding domain but to higher values in neighboring domains. This behavior can be observed in all three sensitivity maps. Comparing the sensitivity coefficients of GM and WM, a significant mutual interference can be observed. For example, a change in the electrical conductivity in WM also greatly affects the resulting electric field in GM and vice versa. A comparison between the magnitudes of the sensitivity coefficients emphasizes the need for more exact knowledge about the electrical conductivity of GM that can be explained by its embedded geometrical position between CSF and $\mathrm{WM}$ as well as its high range in uncertainty in the input.

\section{CONCLUSION}

A gPC expansion using two different approaches to determine the gPC coefficients, namely, quadrature and regression, is compared with an SC in the framework of TMS. No significant advantages or disadvantages between the methods could be observed in the present framework of tensored grids. An investigation regarding the convergence properties of these methods revealed an acceptable accuracy with errors $<1 \%$ (Fig. 3) regarding the mean, the variance, and the global sensitivity coefficients of the induced electric field using an order $p=4$ expansion.

The results indicate the major influence of the uncertainty in electrical conductivity on the induced electric field. The spatial distribution of the statistical moments as well as the global sensitivity coefficients contribute to a better understanding about uncertainty propagation in TMS. The sensitivity analysis shows that the electrical conductivity of GM plays the largest role in the whole process. The relative standard deviation reveals that the electric field in the vicinity of a cortical sulcus is affected most by the uncertainty. Besides geometrical variations between patients, the uncertainty of electrical conductivity could be a key factor by explaining the variability between clinical TMS studies.

The advantage of a simplified model, such as the sulcus/gyrus structure employed in this paper, lies in its practicality due to computational cost and, hence, its applicability for parametric studies. The simplified structure allows the interpretation of data and the explanation of underlying processes. However, in order to quantify the uncertainty in TMS, realistic head models have to be used in combination with the presented methodology. These studies require highly efficient implementations due to increased computational costs. This could imply the application of either Smolyak-based sparse grids [24], [35] for approaches based on Gauss quadrature and SC or reduced grids in the case of regression [36]. The use of adaptive strategies, i.e., proposed in [37] and [38], are very efficient considering one random variable. However, in the case of multiple random output variables, such as in the case of random vector fields, adaptive strategies based on nonintrusive approaches are still in development.

The presented methodology provides valuable insight into the system under investigation. By means of statistical and sensitivity analysis, it is possible to provide more general answers from a numerical perspective.

This paper demonstrates how gPC- and SC-based algorithms could find their application in the field of transcranial stimulation. The presented approach is not limited to TMS; it can be readily applied to other stimulation methods, such as transcranial direct current stimulation [39], to investigate the propagation of uncertainty throughout a particular model under investigation onto respective output quantities. For example, one field of application of the presented schemes could be the evaluation and minimization of the risk to benefit ratio across individuals. The variability can be quantified while considering a constant external stimulation dose [40]. Studies that employ numerical simulations to investigate patient safety of new systems or coil design and optimization could also benefit from approaches based on spectral projection [41]. The simple sulcus/gyrus model presented here demonstrates the impact of uncertainty and necessity of this kind of study in the broad framework of brain stimulation.

\section{ACKNOWLEDGMENT}

This work was supported by the Deutsche Forschungsgemeinschaft in the framework of the Research Training Group 1567 at the Technische Universitaet Ilmenau, Germany. The authors would like to thank L. Codecasa for his support and many helpful suggestions.

\section{REFERENCES}

[1] A. T. Barker, R. Jalinous, and I. L. Freeston, "Non-invasive magnetic stimulation of human motor cortex," Lancet, vol. 1, no. 8437, pp. 1106-1107, 1985.

[2] A. T. Barker, "An introduction to the basic principles of magnetic nerve stimulation," J. Clin. Neurophysiol., vol. 8, no. 1, pp. 26-37, 1991.

[3] Z.-D. Deng, S. H. Lisanby, and A. V. Peterchev, "Electric field depthfocality tradeoff in transcranial magnetic stimulation: Simulation comparison of 50 coil designs," Brain Stimul., vol. 6, no. 1, pp. 1-13, 2013.

[4] L. Hernandez-Garcia, T. Hall, L. Gomez, and E. Michielssen, "A numerically optimized active shield for improved transcranial magnetic stimulation targeting," Brain Stimul., vol. 3, no. 4, pp. 218-225, 2010.

[5] A. Thielscher, A. Opitz, and M. Windhoff, "Impact of the gyral geometry on the electric field induced by transcranial magnetic stimulation," Neurolmage, vol. 54, no. 1, pp. 234-243, 2011.

[6] L. A. Geddes and L. E. Baker, "The specific resistance of biological material-A compendium of data for the biomedical engineer and physiologist," Med. Biol. Eng., vol. 5, no. 3, pp. 271-293, 1967.

[7] C. Gabriel, S. Gabriel, and E. Corthout, "The dielectric properties of biological tissues: I. Literature survey," Phys. Med. Biol., vol. 41, no. 11, pp. 2231-2249, 1996.

[8] L. J. Gomez, A. C. Yucel, L. Hernandez-Garcia, S. F. Taylor, and E. Michielsson, "Uncertainty quantification in transcranial magnetic stimulation via high-dimensional model representation," IEEE Trans. Biomed. Eng., vol. 61, no. 1, pp. 361-372, Jan. 2015.

[9] S. Silva, P. J. Basser, and P. C. Miranda, "Elucidating the mechanisms and loci of neuronal excitation by transcranial magnetic stimulation using a finite element model of a cortical sulcus," Clin. Neurophysiol., vol. 119, no. 10, pp. 2405-2413, 2008. 
[10] R. Salvador, S. Silva, P. J. Basser, and P. C. Miranda, "Determining which mechanisms lead to activation in the motor cortex: A modeling study of transcranial magnetic stimulation using realistic stimulus waveforms and sulcal geometry," Clin. Neurophysiol., vol. 122, no. 4, pp. 748-758, 2011.

[11] COMSOL Multiphysics Version 4.4., COMSOL, Inc., Burlington, MA, USA, 2014

[12] R. G. Ghanem, Stochastic Finite Elements: A Spectral Approach. New York, NY, USA: Springer-Verlag, 2012.

[13] L. Codecasa and L. Di Rienzo, "Stochastic finite integration technique formulation for electrokinetics," IEEE Trans. Magn., vol. 50, no. 2, Feb. 2014, Art. ID 7014104.

[14] L. Codecasa and L. Di Rienzo, "Generalized spectral decomposition approach to a stochastic finite integration technique electrokinetic formulation," in Proc. 9th IET Int. Conf. Comput. Electromagn., London, U.K., Mar./Apr. 2014, pp. 1-2.

[15] R. Gaignaire, R. Scorretti, R. V. Sabariego, and C. Geuzaine, "Stochastic uncertainty quantification of eddy currents in the human body by polynomial chaos decomposition," IEEE Trans. Magn., vol. 48, no. 2, pp. 451-454, Feb. 2012.

[16] S. Gabriel, R. W. Lau, and C. Gabriel, "The dielectric properties of biological tissues: III. Parametric models for the dielectric spectrum of tissues," Phys. Med. Biol., vol. 41, no. 11, pp. 2271-2293, 1996.

[17] J. Latikka, T. Kuurne, and H. Eskola, "Conductivity of living intracranial tissues," Phys. Med. Biol., vol. 46, no. 6, pp. 1611-1616, 2001.

[18] D. Güllmar, J. Haueisen, and J. R. Reichenbach, "Influence of anisotropic electrical conductivity in white matter tissue on the EEG/MEG forward and inverse solution. A high-resolution whole head simulation study," NeuroImage, vol. 51, no. 1, pp. 145-163, 2010.

[19] R. Salvador, F. Ramirez, M. V'yacheslavovna, and P. C. Miranda, "Effects of tissue dielectric properties on the electric field induced in tDCS: A sensitivity analysis," in Proc. 34th Annu. Int. Conf. IEEE Eng. Med. Biol. Soc. (EMBC), Aug./Sep. 2012, pp. 787-790.

[20] E. Wassermann, C. Epstein, V. Walsh, T. Paus, S. Lisanby, and U. Ziemann, The Oxford Handbook of Transcranial Stimulation (Oxford Handbooks). Oxford, U.K.: Oxford Univ. Press, 2008.

[21] H. S. Suh, W. H. Lee, and T.-S. Kim, "Influence of anisotropic conductivity in the skull and white matter on transcranial direct current stimulation via an anatomically realistic finite element head model,' Phys. Med. Biol., vol. 57, no. 21, pp. 6961-6980, 2012.

[22] D. Xiu, Numerical Methods for Stochastic Computations: A Spectral Method Approach. Princeton, NJ, USA: Princeton Univ. Press, 2010.

[23] R. Ghanem and P. D. Spanos, Stochastic Finite Elements: A Spectral Approach. Minneola, NY, USA: Dover, 2003.

[24] O. Le Maitre and O. M. Knio, Spectral Methods for Uncertainty Quantification. Dordrecht, The Netherlands: Springer-Verlag, 2010.
[25] D. Xiu and J. S. Hesthaven, "High-order collocation methods for differential equations with random inputs," SIAM J. Sci. Comput., vol. 27, no. 3, pp. 1118-1139, 2005.

[26] M. D. Mckay, R. J. Beckman, and W. J. Conover, "A comparison of three methods for selecting values of input variables in the analysis of output from a computer code," Technometrics, vol. 42, no. 1, pp. 55-61, 2000.

[27] W. J. Morokoff and R. E. Caflisch, "Quasi-Monte Carlo integration," J. Comput. Phys., vol. 122, no. 2, pp. 218-230, 1995.

[28] L. Plaskota and H. Woźniakowski, Monte Carlo and Quasi-Monte Carlo Methods 2010, vol. 23. Berlin, Germany: Springer-Verlag, 2012.

[29] D. Xiu, "Fast numerical methods for stochastic computations: A review," Commun. Comput. Phys., vol. 5, nos. 2-4, pp. 242-272, 2009.

[30] R. J. Renka, "Multivariate interpolation of large sets of scattered data," ACM Trans. Math. Softw., vol. 14, no. 2, pp. 139-148, 1988.

[31] R. V. Field and M. Grigoriu, "On the accuracy of the polynomial chaos approximation," Probab. Eng. Mech., vol. 19, nos. 1-2, pp. 65-80, 2004.

[32] S. S. Wilks, "Certain generalizations in the analysis of variance," Biometrika, vol. 24, nos. 3-4, pp. 471-494, 1932.

[33] R. J. Freund, W. J. Wilson, and P. Sa, Regression Analysis: Statistical Modeling of a Response Variable, 2nd ed. Burlington, MA, USA: Elsevier, 2006.

[34] F. Pukelsheim, Optimal Design of Experiments (Classics in Applied Mathematics), vol. 50. Philadelphia, PA, USA: SIAM, 2006.

[35] S. A. Smolyak, "Quadrature and interpolation formulas for tensor products of certain classes of functions," Soviet Math., Doklady, vol. 4, no. 1, pp. 240-243, 1963.

[36] B. Sudret, "Global sensitivity analysis using polynomial chaos expansions,” Rel. Eng. Syst. Safety, vol. 93, no. 7, pp. 964-979, 2008.

[37] G. Blatman and B. Sudret, "Sparse polynomial chaos expansions and adaptive stochastic finite elements using a regression approach," Comp. Rendus Mécanique, vol. 336, no. 6, pp. 518-523, 2008.

[38] G. Blatman and B. Sudret, "An adaptive algorithm to build up sparse polynomial chaos expansions for stochastic finite element analysis," Probab. Eng. Mech., vol. 25, no. 2, pp. 183-197, 2010.

[39] M. A. Nitsche et al., "Transcranial direct current stimulation: State of the art 2008," Brain Stimul., vol. 1, no. 3, pp. 206-223, 2008.

[40] A. V. Peterchev et al., "Fundamentals of transcranial electric and magnetic stimulation dose: Definition, selection, and reporting practices," Brain Stimul., vol. 5, no. 4, pp. 435-453, 2012.

[41] P. A. Muller, A. Pascual-Leone, and A. Rotenberg, "Safety and tolerability of repetitive transcranial magnetic stimulation in patients with pathologic positive sensory phenomena: A review of literature," Brain Stimul., vol. 5, no. 3, pp. 320-329.e27, 2012. 